\title{
Mathematical modelling and numerical simulation of linear electro-hydraulic servomechanism with stepper motor
}

\author{
Vergil Muraru, ${ }^{1, *}$, Constantin Calinoiu ${ }^{2}$, Sebastian Muraru ${ }^{1}$, Ana Dulgheru ${ }^{1}$ and Cornelia \\ Muraru-Ionel $^{1}$ \\ ${ }^{1}$ INMA Bucharest, CAE Department, 013811, Bucharest, Romania \\ ${ }^{2}$ Politehnica University from Bucharest, Power Faculty, 060042, Bucharest, Romania
}

\begin{abstract}
The paper presents the results of research on the performance of linear electro-hydraulic servomechanisms with stepper motor, evaluated by mathematical modelling and numerical simulation with the AMESIM software package. These servomechanisms have a great potential for application, including for agricultural technical equipment. Servomechanisms of various types are used for the rapid and high precision operation of the various systems. In practice, electro-hydraulic servomechanisms have been required in the case of the systems requiring high forces or moments. These equipment are automatic hydraulic tracking systems and can be analysed with methods specific to automatic systems. The paper contains a structural description of an electro-mechanical servomechanism with stepper motor, its operation, mathematical model and its performances obtained by numerical simulation. The mathematical modelling of the electro-hydraulic servomechanism is based on the relations between the input sizes and output sizes of the equipment in its structure as well as the connection relations between these equipment. Based on the obtained results it is demonstrated that the analysis and synthesis of electro-hydraulic servomechanisms with stepper motor can be determined by numerical simulation using the AMESIM simulation software.
\end{abstract}

\section{Introduction}

A linear electrohydraulic servomechanism is an automatic tracking system that achieves the transformation of the input size of electrical nature into a linear displacement (output size), developing also simultaneously a high output force.

The elements from the structure of a typical linear electro-hydraulic servomechanism are: an electrohydraulic amplifier, a hydraulic cylinder, a displacement transducer whose output signal constitutes the reaction link of the servomechanism and a servo controller (electronic block) [1].

\footnotetext{
* Corresponding author: virgil.muraru@gmail.com
} 
The performances of the electrohydraulic servomechanisms are dependent on the performances of the electrohydraulic amplifiers in their structure.

The electrohydraulic amplifier has in its structure an electromechanical converter and an electrohydraulic converter (hydraulic distributor) [2].

The electromechanical converter is an equipment that has as an input signal an electrical size, and at its output it has a displacement or a force that is proportional to the input size.

The shape, dimensions and performances of the electrohydraulic amplifier are dependent on the type of electromechanical converter from its structure [3]. In practice, the following types of electromechanical converters were imposed:
a) converter with permanent magnet and movable coil (Fig. 1);
b) converter with permanent magnets and fixed coils ("torque motor") (Fig.2);
c) proportional electromagnet (fig. 3);
d) linear motor force (fig. 4);
e) stepper motor (fig.5).
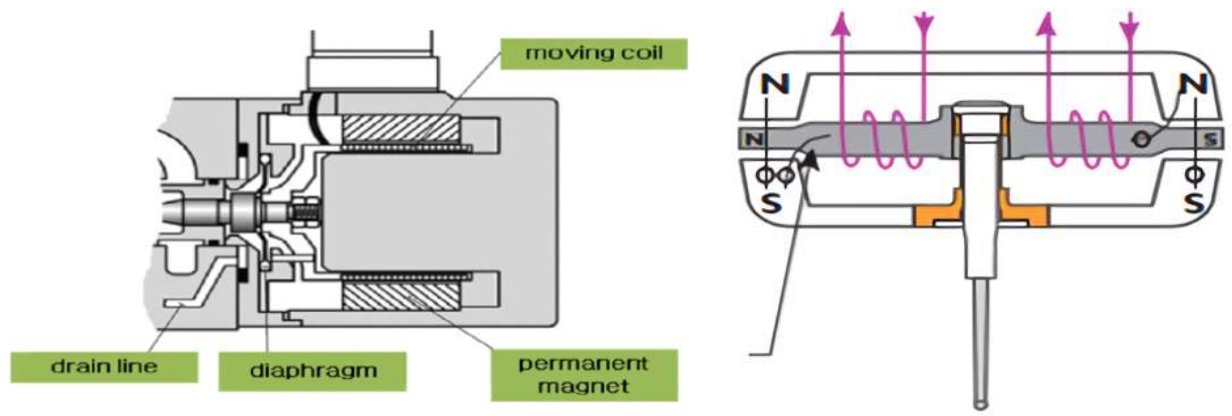

Fig. 1. Movable coil

Fig. 2. Torque motor
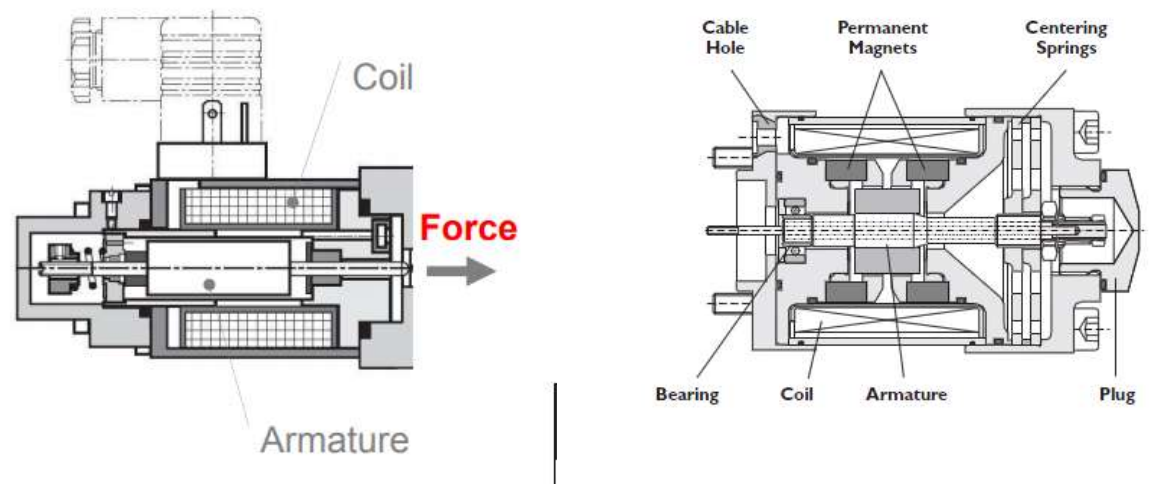

Fig. 3. Proportional magnet

Fig. 4. Linear force motor

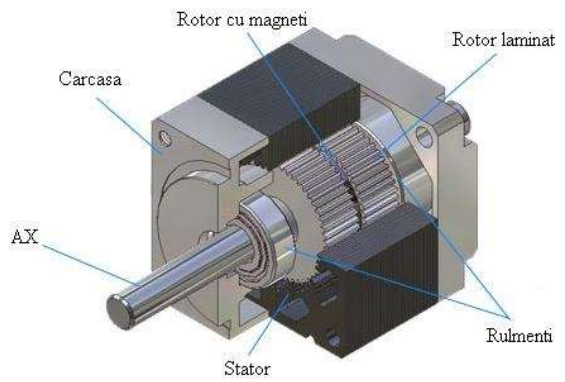

Fig. 5. Stepper motor 
In the case of electrohydraulic servomechanisms, the Hydro-Mechanical Converter has the shape of a hydraulic distributor that transforms the input size of mechanical nature (displacement) into a hydraulic size (flow rate / debit).

The electrohydraulic servomechanisms with stepper motor do not have in their structure a displacement transducer for achieving the reaction link, their reaction size is of mechanical nature, and it is realized by means of a screw-nut mechanism.

Consequently, the servomechanism with stepper motor is a servomechanism which has the input of electric nature, the output of mechanical nature and the reaction size of mechanical nature.

\section{Structure and operation}

Figure 6 presents the principle diagram of an electrohydraulic servomechanism that has in its structure an electrohydraulic amplifier achieved with an electric stepper motor [4].

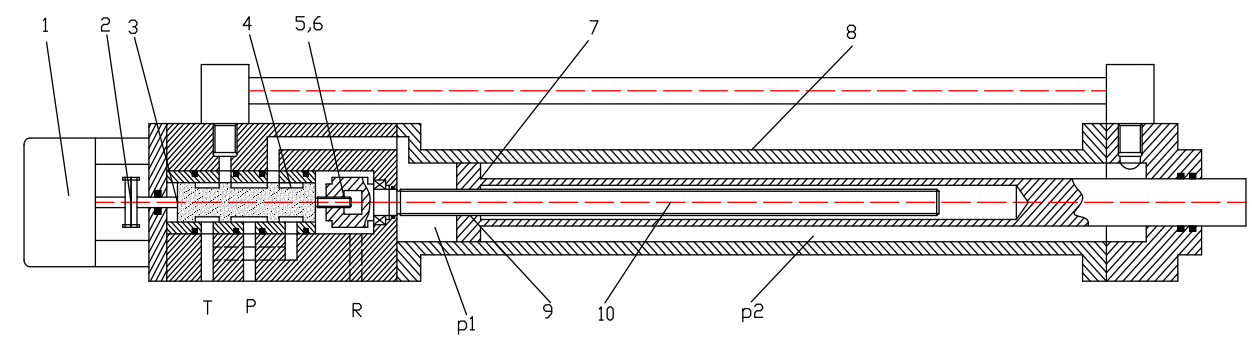

Fig. 6. Electrohydraulic servomechanism with stepper motor

The angular displacement of the shaft of the electric motor 1 is transformed into a linear displacement by means of the elastic coupling 2 and of the screw-nut assembly 5 . When the motor shaft rotates with the prescribed angle $\boldsymbol{\theta}$, the screw 5 from the end of the rod 3 advances into the nut 6 , which is temporarily fixed.

The axial displacement of the screw is possible because the elastic coupling 2 may deform axially, this being achieved in the shape of two membranes embedded on the contour.

The spool 4 is connected to the rod 3 through a spring (not shown on the drawing). The linear displacement of the rod is also transmitted to the spool. The spool is endowed with a rotational movement locking system, so that, it can only move linearly simultaneously with the rod.

When the motor shaft moves clockwise, the rod advances into the nut, the spool moves to the right, the distributor opens and the pressurized oil reaches the chamber from the left side of the hydraulic cylinder 8 .

Simultaneously, the chamber from the right side of the hydraulic cylinder is connected to the tank. Under the action of the pressure forces, the piston 7 will move to the right with the value " $\mathrm{z}$ " (the cylinder rod is moving in the outward direction).

In the piston, a lead nut 9 is achieved, that has the sense of the screw thread, opposite to the direction of the nut 6 .

When the lead screw moves linearly, the screw 10 is rotated and also drives the nut 6 , and the screw 5, the rod 3 and implicitly the spool moves linearly in the opposite direction to the initial displacement. The displacement $\mathrm{z}$ of the piston stops when the distributor closes. 
When the engine shaft moves counter clockwise, the pressurized oil reaches the chamber in the right side of the hydraulic cylinder and the output rod moves in the sense of its withdrawal. Thus, a linear relationship can be obtained between the input size $\boldsymbol{\theta}$ and the output size $\mathrm{z}$.

\section{Mathematical modelling}

For the mathematical modelling of the electro-hydraulic servomechanism, it is necessary to write the relations between the input and output sizes of the equipment in its structure, as well as, the connection relations between these equipment [5-6].

\subsection{The characteristic of the step by step electric motor}

Angular displacement of the step by step electric motor shaft is proportional with the control voltage according to the relationship

where:

$$
\theta_{y}=K_{m} \cdot U
$$

- $K_{m}$ - the transfer factor $[\mathrm{rad} / \mathrm{V}]$;

- U - command signal (voltage) [- $(V)]$;

- $\theta_{y}$ - the rotation angle of the shaft $[\mathrm{rad}]$;

\subsection{An equation of the linear displacement of the spool rod}

The angular displacement, $\theta_{y}$, of the stepper motor shaft induces a linear displacement $\mathrm{x} 1$ to the spool driving rod, due to the screw and nut mechanism, according to the next formula:

where

$$
x_{1}=\frac{\theta_{y}}{2 \pi} p_{s r}
$$

- $\mathrm{x}_{1}$ - spool rod displacement $[\mathrm{m}]$;

- $p_{s r}$ - reaction screw step $[m]$.

\subsection{The equations of angular displacement of the screw and linear displacement of the spool rod}

The lead screw has an angular displacement when the cylinder piston moves linearly, due to the advance screw and nut mechanism, according to the next formula:

$$
\theta_{z}=\frac{2 \pi}{p_{s a}} z
$$

where:

- $p_{s a}$ - lead screw step, $[\mathrm{m}]$;

- $\mathrm{z}$ - hydraulic cylinder piston displacement, $[\mathrm{m}]$.

- $\theta_{z}$ - screw rotation angle, $[\mathrm{rad}]$.

The lead screw also induces the nut the same angular displacement $\theta_{z}$ and this induces a linear displacement to the spool rod according to the other formula: 
where

$$
x_{2}=\frac{\theta_{z}}{2 \pi} p_{s r}=\frac{z}{p_{s a}} p_{s r}
$$

- $\mathrm{x}_{2}$ - spool rod displacement [m].

\subsection{Comparator equation}

The opening of the distributor is equal to the displacement of the spool, according to the next formulas:

$$
x=x_{1}-x_{2}=\left(\frac{\theta_{y}}{2 \pi}-\frac{z}{p_{s a}}\right) p_{s r}
$$

or

$$
x=x_{1}-x_{2}=\left(\frac{K_{m} \cdot U}{2 \pi}-\frac{z}{p_{s a}}\right) p_{s r}
$$

\subsection{Piston displacement equation}

$$
A_{p 1} \cdot p_{1}-A_{p 2} \cdot p_{2}=m \cdot \ddot{z}+k_{f} \cdot \dot{z}+k_{e} \cdot z+F_{v}
$$

where:

- $\mathrm{A}_{\mathrm{p} 1}, \mathrm{~A}_{\mathrm{p} 2}$ - the frontal areas of the piston, $\left[\mathrm{m}^{2}\right]$;

- $\mathrm{p} 1, \mathrm{p} 1$ - the pressures in the cylinder chambers, $[\mathrm{Pa}]$;

- $\mathrm{m}$ - the equivalent mass of the actuated system reduced to the piston rod, $[\mathrm{kg}]$;

- z, $\dot{Z}, \ddot{Z}$ - displacement, speed and acceleration of the piston, $[\mathrm{m}],[\mathrm{m} / \mathrm{s}],\left[\mathrm{m} / \mathrm{s}^{2}\right]$;

- $k_{f}$ - viscous friction coefficient $[N s / m]$;

- $k_{e}$ - elastic force coefficient $[\mathrm{N} / \mathrm{m}]$;

- $F_{v}$ - disruptive force $[N]$.

\subsection{Continuity equation corresponding to the hydraulic cylinder}

The continuity equation corresponding to the hydraulic cylinder has the next form:

where:

$$
\begin{aligned}
& Q_{1}=A_{p 1} \frac{d z}{d t}+c_{i p} \cdot\left(p_{1}-p_{2}\right)+c_{e p} \cdot p_{1}+\frac{V_{1}}{E} \frac{d p_{1}}{d t} \\
& Q_{2}=A_{p 2} \frac{d z}{d t}+c_{i p} \cdot\left(p_{1}-p_{2}\right)-c_{e p} \cdot p_{2}-\frac{V_{2}}{E} \frac{d p_{2}}{d t}
\end{aligned}
$$

- $\mathrm{Q}_{1}, \mathrm{Q}_{2}$ - the flow rates corresponding to the cylinder chambers $\left[\mathrm{m}^{3} / \mathrm{s}\right]$;

- $\mathrm{c}_{\text {ip }}$ - the internal losses coefficient $\left[\mathrm{m}^{3} / \mathrm{s} . \mathrm{Pa}\right]$;

- $\mathrm{c}_{\mathrm{ep}}$ - the external losses coefficient $\left[\mathrm{m}^{3} / \mathrm{s} . \mathrm{Pa}\right]$;

$-\mathrm{V}_{1}, \mathrm{~V}_{2}$ - cylinder chamber volumes $\left[\mathrm{m}^{3}\right]$.

\subsection{The flow rate equation through orifices}

The general equation for flowing through orifices with sharp edge is: 


$$
Q=c_{d} \cdot A(x) \cdot \sqrt{\frac{2 \Delta p}{\rho}}
$$

where:

- Q - the debit transited through the orifice $\left[\mathrm{m}^{3} / \mathrm{s}\right]$;

$-c_{d}-$ the orifice flow coefficient [-];

- $\mathrm{A}(\mathrm{x})$ - the orifice area $\left[m^{2}\right]$;

- $\Delta \mathrm{p}$ - pressure drop on the orifice $[\mathrm{Pa}]$;

- $\rho$ - the fluid density $\left[\mathrm{kg} / \mathrm{m}^{3}\right]$.

In the concrete situation of the servomechanism, this equation has a particular form:

For $x \geq 0$

$$
\begin{gathered}
Q_{1}=c_{d} \cdot A(x) \cdot \sqrt{\frac{2\left(p_{s}-p_{1}\right)}{\rho}} \\
Q_{2}=c_{d} \cdot A(x) \cdot \sqrt{\frac{2 p_{2}}{\rho}}
\end{gathered}
$$

for $x<0$

$$
\begin{gathered}
Q_{1}=c_{d} \cdot A(x) \cdot \sqrt{\frac{2 p_{1}}{\rho}} \\
Q_{2}=c_{d} \cdot A(x) \cdot \sqrt{\frac{2\left(p_{s}-p_{2}\right)}{\rho}}
\end{gathered}
$$

\section{Results and discussions}

\subsection{Calculation of the servomechanism resolution}

The servomechanism resolution is dependent on the value of the stepper motor resolution $\Delta \boldsymbol{\theta}$ and the lead screw step value $\mathrm{p}_{s a}$ and is calculated with the next formula:

The value $\Delta \boldsymbol{\theta}$ is calculated with the formula

$$
\operatorname{Rez}=\frac{\Delta \theta \cdot p_{s a}}{2 \pi}[\mathrm{mm}]
$$

$$
\Delta \theta=\frac{2 \pi}{N p}
$$

Where $N p$ represents the number of steps of the motor for a complete rotation.

Table 1 shows the servo mechanical resolution values for different values of the motor resolution and different values of the lead screw step.

Table 1. Servomechanism resolution

\begin{tabular}{|c|c|c|c|}
\hline \multirow{2}{*}{$\begin{array}{c}\text { psa } \\
{[\mathrm{mm}]}\end{array}$} & \multicolumn{3}{|c|}{ The number of motor steps per rotation } \\
\cline { 2 - 4 } & 100 & 200 & 400 \\
\cline { 2 - 4 } & \multicolumn{3}{|c|}{ Resolution $[\mu \mathrm{m}]$} \\
\hline 4 & 40 & 20 & 10 \\
\hline 6 & 60 & 30 & 15 \\
\hline 8 & 80 & 40 & 20 \\
\hline
\end{tabular}




\subsection{Numerical simulation}

Figure 7 shows the block diagram of the servomechanism

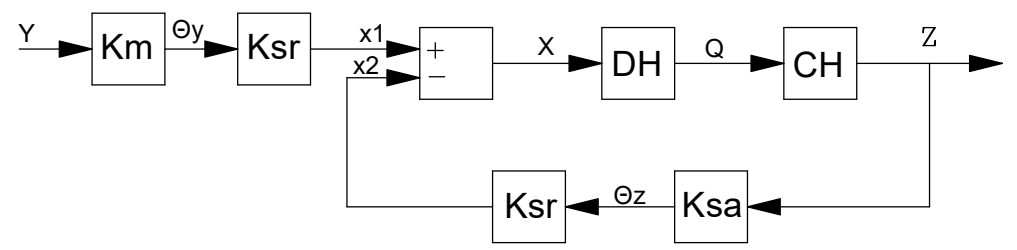

Fig. 7. Block diagram of the stepper servomechanism

For the numerical simulation of the servomechanism operation, the AMESIM software package was used [7-8]. The scheme of the simulation network is shown in Fig. 7.

The numerical simulation was performed for the following numerical values of the parameters: Piston diameter $\mathrm{D}=50 \mathrm{~mm}$, Rod diameter $\mathrm{d}=35 \mathrm{~mm}$,

The total piston stroke $\mathrm{h}=500 \mathrm{~mm}$, the supply pressure Ps $=200 \mathrm{bar}$, the reaction screw transfer factor $\mathrm{Ksr}=2 \pi \mathrm{rad} / \mathrm{mm}$, the lead screw transfer factor Ksa $=4 \mathrm{~mm} / 2 \pi \mathrm{rad}$, the

For easiness of the results interpretation, the input size $\mathrm{Y}$ has been entered in digital form having millimeter as measuring unit.

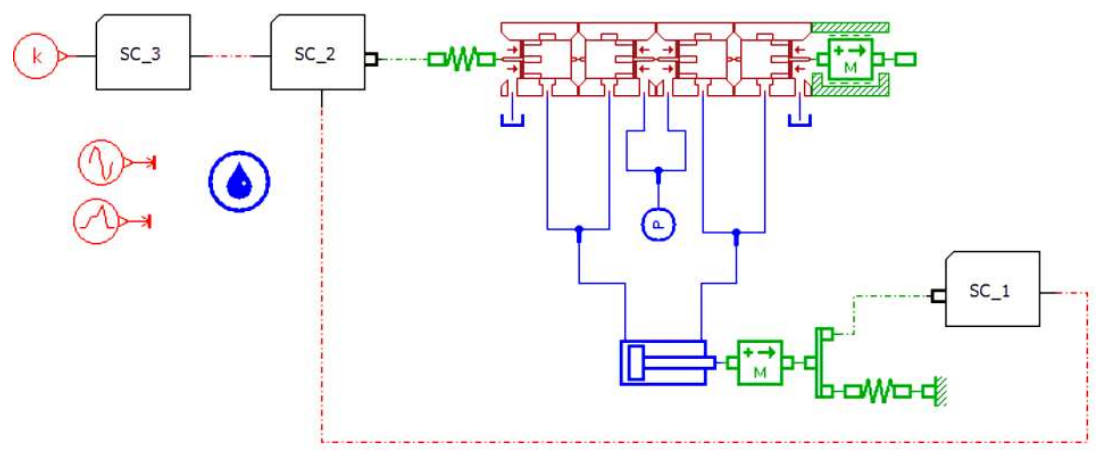

Fig. 8. The servomechanism simulation network

The results of the numerical simulations are presented synthetically in the form of diagrams.

In Figures 9, the numerically simulated command signal is presented and in Figure 10, the response of the servomechanism to the sinusoidal signal is shown.

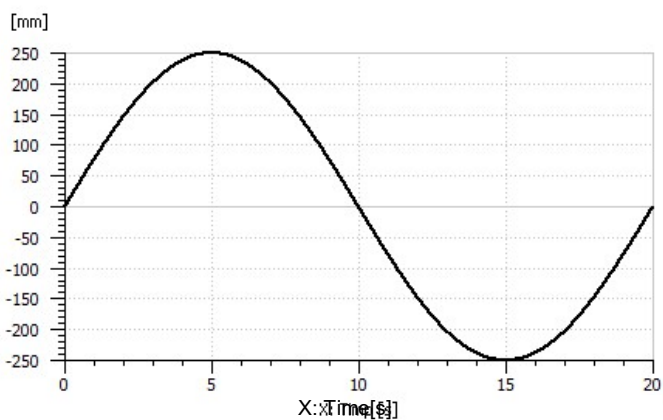

Fig. 9. The numerically simulated command signal

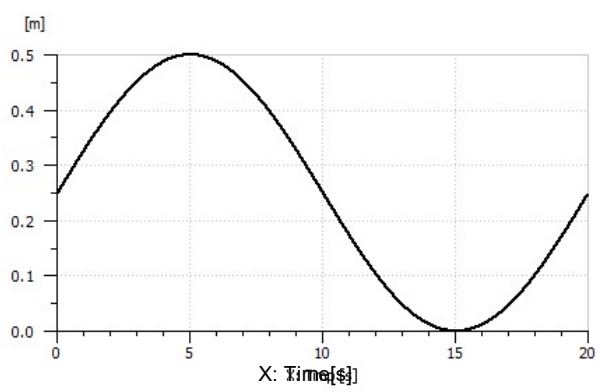

Fig. 10. Piston displacement 
The servomechanism static characteristic is shown in Figure 11 and in Figure 12 is shown the variation in time of the pressures in the cylinder chambers when it is controlled with a sinusoidal signal.

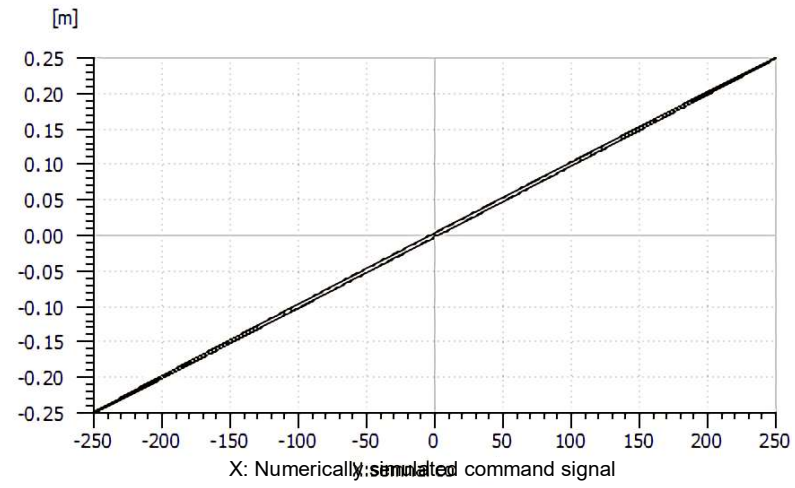

Fig. 11. The servomechanism static characteristic

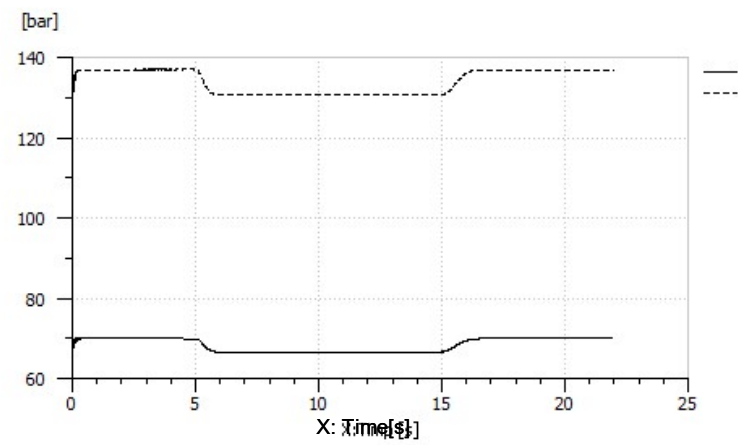

Fig. 12. Variation in time of the pressures in the servomechanism chambers. (continuous line pressure in chamber 1, dashed line - pressure in chamber 2.

Figure 13 shows the hysteresis characteristic of the servomechanism for the situation in which the distribution windows of the distributor are equal.

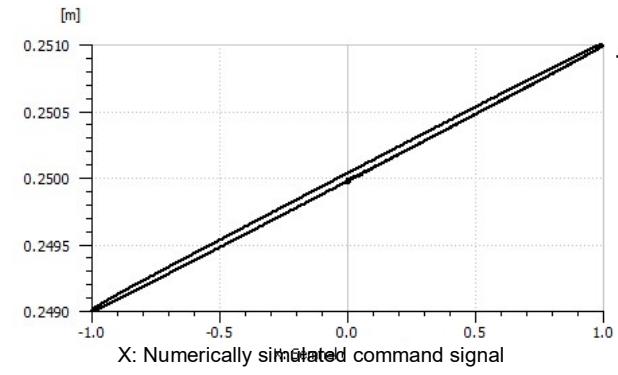

a)

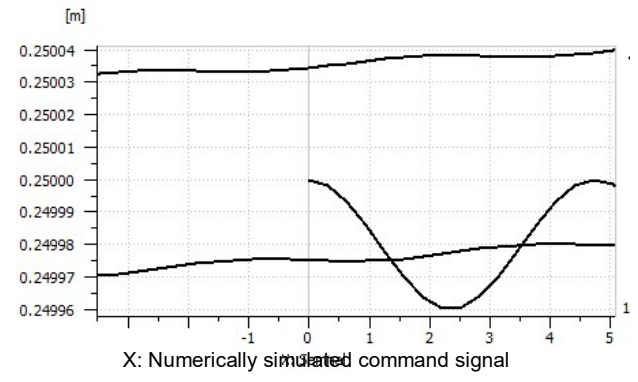

b)

Fig. 13 a) Hysteresis characteristic for distributor with equal distributed windows. b) Detail

Figure 14 shows the hysteresis characteristic of the servomechanism for the situation in which the distribution windows of the distributor are unequal. 


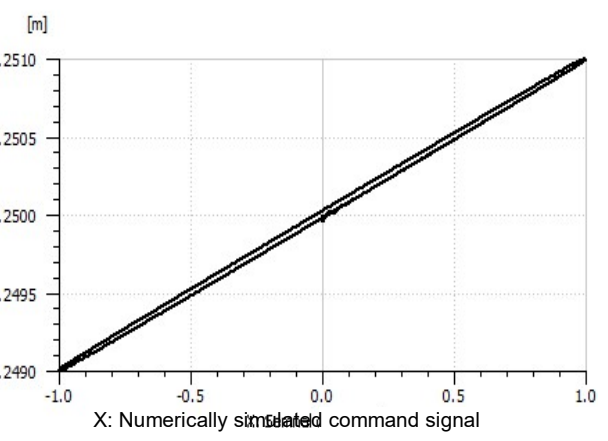

a) [m]

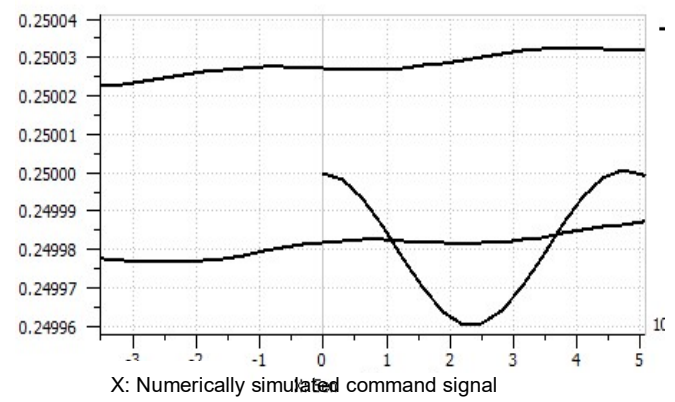

b)

Fig. 14. a) Hysteresis characteristic for distributor with unequal distribution windows., b) Detail

Figure 15 shows the response of the servomechanism to the step type signal

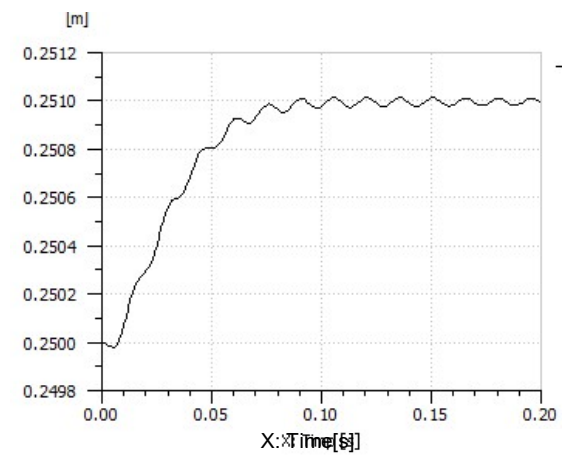

a) Piston displacement

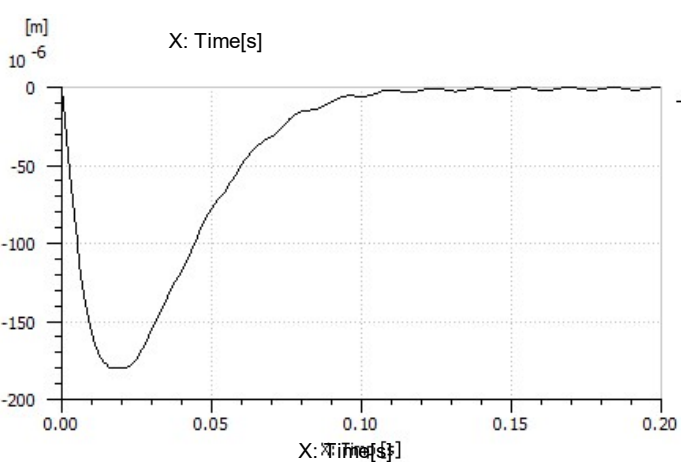

b) Spool displacement

Fig.15. The servomechanism response to step type signal

Figure 16 shows the evolution of the error and of the flow rates corresponding to the two chambers of the servomechanism during the transient regime, when the servomechanism was excited with a step type signal.

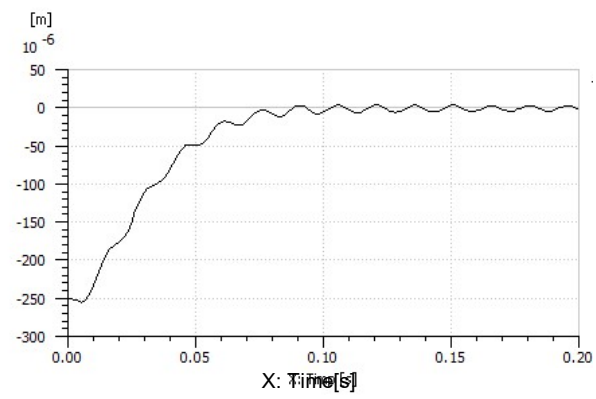

a) error

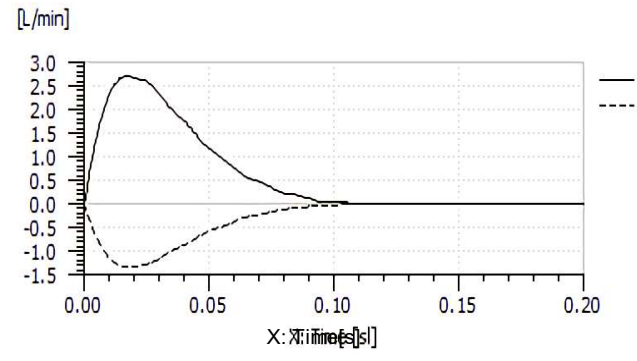

b) flow rates

Fig.16. Variation of error and flow rates in the servomechanism chambers during the transient regime (step type excitation signal) 


\section{Conclusions}

The analysis and the synthesis of electro-hydraulic servomechanisms with stepper motor can be determined by mathematical modelling and numerical simulation using the AMESIM simulation program.

Regarding the servomechanism performances evaluated by mathematical modelling and numerical simulation with AMESIM software, the following conclusions were established:

1) The time constant of the servomechanism is $0.03 \mathrm{~s}$.

2) The resolution of the servomechanism is significantly influenced by the resolution of the stepper motor and the lead screw step.

3) The servomechanism hysteresis value is dependent on the type of distribution windows of the distributor. Because the hydraulic cylinder has the areas of the piston unequal, the hysteresis value is lower when the distribution window areas are unequal.

4) The lower value of the hysteresis was obtained for the ratio of the distributor slots areas that is equal to the piston areas ratio. For equal areas, the hysteresis is with 25 microns higher under the same operating conditions.

This work was supported by a grant of the Romanian Education and Research Ministry (RRIM), through Programme 1 - Development of the national research-development system, subprogram 1.2 Institutional performance - Projects for financing excellence in RDI, contract no. 16PFE, and was done by "NUCLEU" Programme, developed with the support of the RRIM, project PN 19100102.

\section{References}

1. N. Vasiliu, D. Vasiliu, Hydraulic and pneumatic drives (Technical Publishing House, 2008)

2. H.E. Merritt, Hydraulic Control Systems (John Wiley and Sons Inc., New York, London, Sydney, 1967)

3. *** Technical documentation of MOOG company

4. $* * *$ Technical documentation of CURTISS -WRIGHT company

5. C. Călinoiu, N. Vasiliu, D. Vasiliu, I. Catană, Modeling, simulation and identification of hydraulic servomechanisms (Technical Publishing House, 1998)

6. I. Ursu, F. Ursu, Active and semiactive control (Romanian Academy Publishing House, 2002)

7. *** LMS INTERNATIONAL. Advanced Modelling and Simulation Environment, (Release 13 User Manual, Leuven, 2013)

8. N. Vasiliu, D. Vasiliu, C. Calinoiu, R. Puhalschi, Simulațion of fluid power Systems with LMS Imagine (Lab Amesim CCR Press Publishing House, 2018) 